\title{
Web Based Visualization of Digital Mock-Up with Standardized 3D Formats
}

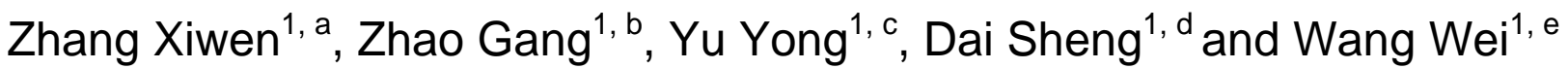 \\ ${ }^{1}$ School of Mechanical Engineering and Automation, Beijing University of Aeronautics and \\ Astronautics, Beijing 100191, China; \\ azhangxiwen@buaa.edu.cn, bzhaog@buaa.edu.cn, cyuyong@buaa.edu.cn, \\ ddaisheng@buaa.edu.cn, ejrrt@buaa.edu.cn
}

Keywords: Engineering Visualization, Digital Mock-Up, JT Standard, Web.

\begin{abstract}
Nowadays, parts of complex products are usually developed by different suppliers distributed all over the world. To share the 3D model timely and improve collaboration efficiency among the business partners, it is necessary to support the web based visualization of DMU. Among the widely used standardized formats, JT format has many special advantages and has been standardized as ISO 14306 “JT file format specification for 3D visualization". This paper introduces the JT Standard and provides a novel method to implement the web based visualization of DMU JT format. A case from the aviation industry using this method is given at length.
\end{abstract}

\section{Introduction}

The increasing complexity of product development and manufacturing processes require global collaboration of designing and manufacturing teams in different enterprises, which involves massive cross-platform \& interdisciplinary data that is changing frequently [1,2]. Dealing with the large-scale data effectively for a variety of purposes by relevant participators is of extreme importance for the development of complex products.

Digital Mock-Up (DMU) is a public data platform that supports product design and work process control, information transmission and sharing [3]. 3D model of product is established based on DMU [4], which can improve the accessibility to information by different users. Meanwhile the Internet provides an easy and intuitive way to transfer and share information on a basis of cross-platform and cross-zone, thus there is a need to realize the visualization of DMU based on web technology and view the $3 \mathrm{D}$ model in the browser directly in the following respects:

- Designers in different locations can have a quick access to the 3D model through the Internet and retrieve product information synchronously.

- Each of commercial CAD software has its own proprietary format and there are more and more CAD software systems nowadays [5]. Realizing visualization of DMU in the browser directly with neutral 3D formats avoids the process of installing heavy CAD tool packages.

- Not only designers are concerned about 3D model of the product, managers and workers also need to view the model on some occasions. The visualization of DMU based on web allows them to view the model with just a browser and no need for downloading bulks of data.

A method to implement the visualization of DMU in the browser with the standardized 3D visualization format JT is thoroughly introduced in this paper. And based on this method, an example from the aviation industry is demonstrated, showing the feasibility of this approach.

\section{Formats for Visualization in PLM}

PLM, Product Lifecycle Management, is a kind of commercial strategy for enterprise information management, which implements a set of solutions to effectively integrate people, processes and information together. Product Lifecycle Visualization (PLV) is a rising discipline for PLM as the 
demands to share the 3D CAD data with stakeholders who do not have the CAD software and consolidate the CAD data with visualization data from other sources are growing significantly.

Regardless of native CAD formats, users have various 3D formats available for visualization in the engineering field. Using a standardized 3D data format in all downstream processes is a natural and preferable choice for global collaborative engineering. An overall comparison of four prevailing visualization formats can be drawn as shown in Fig. 1 [6-8]. Among these formats JT is most widely used in the automotive, aerospace and various manufacturing industries and it has been standardized as ISO 14306 “JT file format specification for 3D visualization” [9]. Thus JT is selected as the data format for visualization of DMU in this paper.

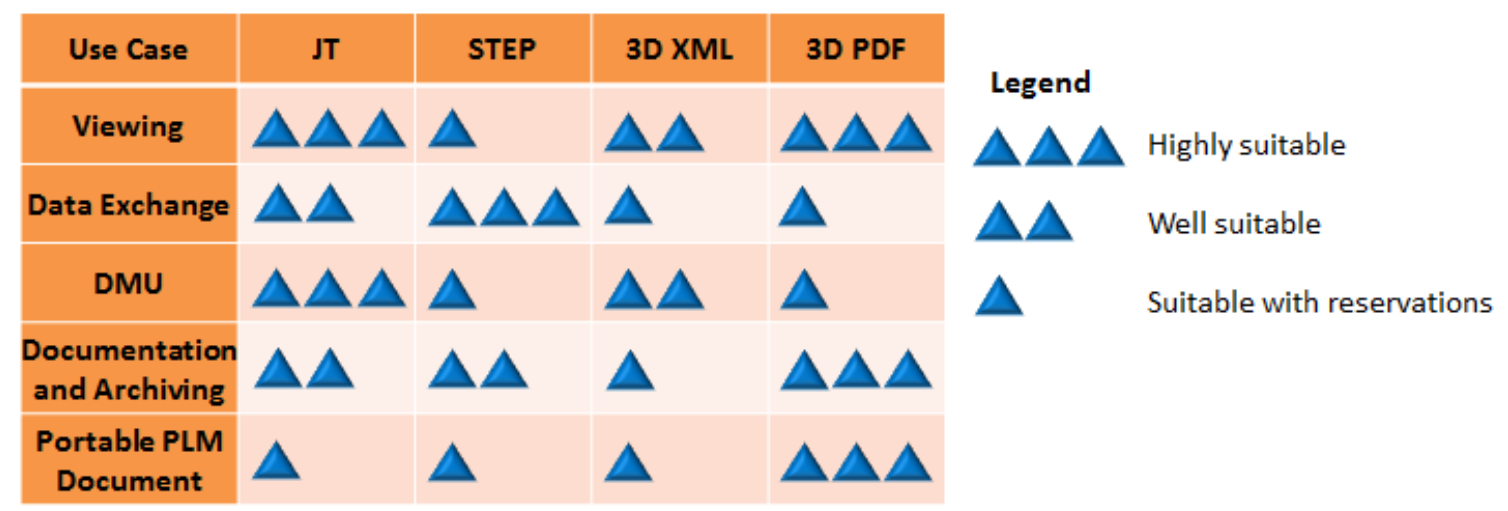

Fig. 1 Comparison of four visualization formats

JT format was first developed by the US company Engineering Animation Inc. in the 1990s, which was taken over by the UGS Corporation subsequently and now is the product of Siemens [10]. As an industrial standard accepted by engineering practices, the JT ISO standardization process begins in the year of 2009 [11].

JT is a binary format and several representations of CAD geometry is supported in its data model. A JT file enables different geometry representations stored in it separately or together [12, 13]:

a) B-REP (Boundary Representation): B-REP data is compressed by using different algorithms and stored without loss, which offers the highest level of representation precision. In the recent specification 10.0, there are two B-REP types permitted: traditional JT B-REP representation and XT B-REP based on the Parasolid boundary representation, which will be favored in the future.

b) Tessellated Geometry: A faceted representation of solids and surfaces is described in Tessellated Geometry. Different levels of detail (LOD), from coarse to fine tessellated representations, can be defined within a JT file. JT files with a low LOD means a lower representation precision but a less volume of data, whereas a high LOD provides an almost exact geometry but the volume of data is large.

c) ULP (Ultra-Lightweight Precise): ULP is a latest compression method. A lightweight, semi-precise representation of the 3D geometry is offered in ULP. The precision of 3D geometry that ULP offers is significantly higher compared with tessellated geometry while the file size is significantly reduced. The main objective is to provide high quality surface geometry with only minor deviations from the original B-REP geometry.

Initially, JT version 8.1 is a publicly available specification (ISO PAS 14306) published by the ISO. The ISO standardization process has been finished by endorsing the ISO standard for JT version 9.5 in 2012 and JT version 10.0 in 2014. ULP and semantic product manufacturing information (PMI, product metadata) are included into the specification.

Due to the advantages of JT compared with other data formats, JT can serve as a standardized, neutral format in nearly all downstream processes, e. g. simulation, digital mock-up and manufacturing [14]. User cases for the application of JT are classified into three categories, related with the complexity of usage: Viewing, Digital Mock-Up Analysis, Design in Context [15]. Viewing is the fundamental use of JT and the utilization of JT in this paper belongs to this case. 


\section{Method of DMU Visualization in the Browser}

JT2Go is a free viewing tool that allows users to view JT model but it is a viewer only for desktop and embedding JT2Go into a web page is not supported. If the JT model can be directly viewed in the browser, the browser will be a collaboration platform for stakeholders in different locations and the 3D model will be shared easily with the help of Internet, which can serve our target to the use of DMU. So the method for using JT as a visualization tool for DMU in browser will be explored in the contents that follow.

\subsection{Generating JT Model}

The first step to realize the visualization of DMU in the browser is to transform 3D model into a standardized 3D data format for visualization after the 3D model created in CAD software. As mentioned above, JT is selected as the data format for visualization of DMU. Most of the CAD software can convert 3D model to JT format, such as UG NX, CATIA and Deep Exploration. UG NX is the product of Siemens and supports the function of setting organization of JT files and LOD from coarse to fine tessellated representations of the JT model, as shown in Fig. 2 and Fig. 3.

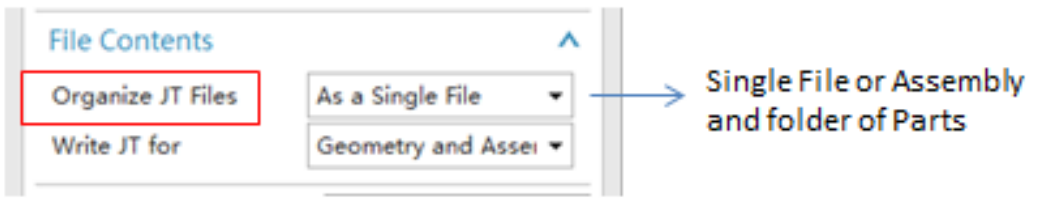

Fig. 2 Set organization of JT files

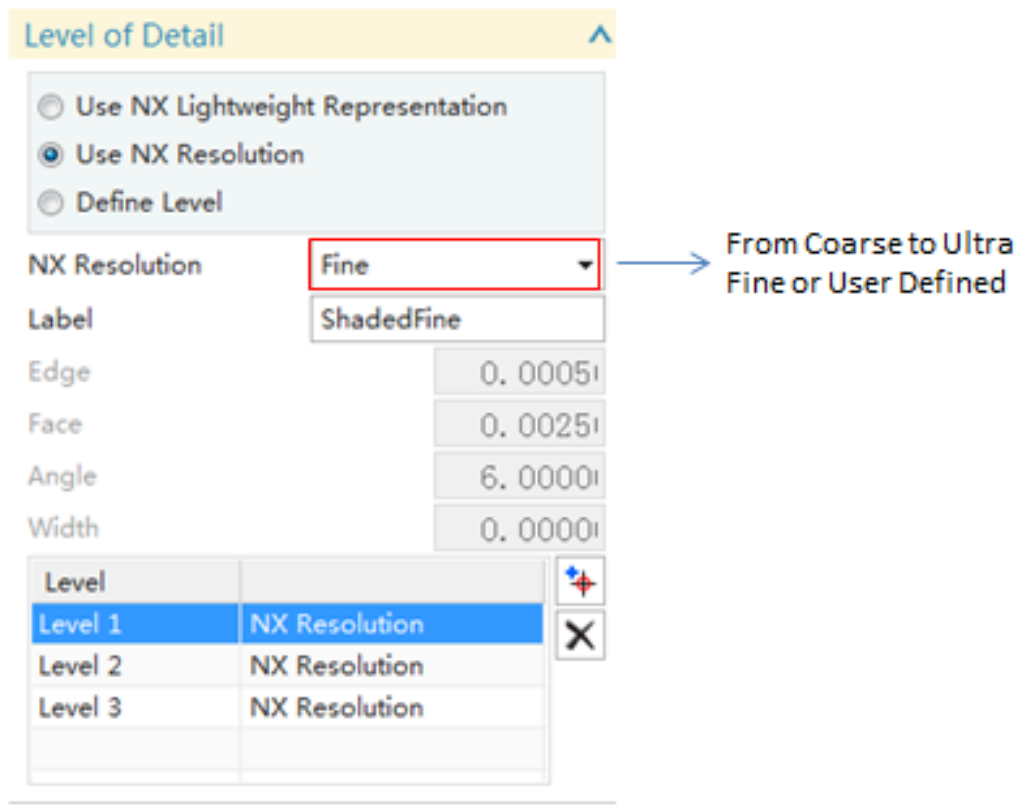

\subsection{Parsing JT Model}

Fig. 3 Set LOD of the JT model

Browser displays text, images and other information in the web and allows user to interact with the information. JT model is hoped to be viewed in the browser to facilitate sharing. To realize this purpose, it is necessary to parse the JT file after exporting the JT model, as the JT format cannot be parsed by browser directly.

According to the ISO 14306 specification, different representations (introduced in section 2) of geometry, product structures and product manufacturing information (PMI) are integrated in a JT file, as shown in Fig. 4. The basic structure of a JT file is presented in Fig. 5, which is divided into three parts. The first part is the File Header, including the basic information of the JT file, such as version information, position information of TOC Segment in JT file, etc. The second part is the TOC Segment, containing information to identify and locate various Data Segment (the third part of JT file). The third part is the Data Segment, which contains various kinds of information related to the product including geometry information, product manufacturing information, etc. 
Standardized Data Format for 3D Visualization

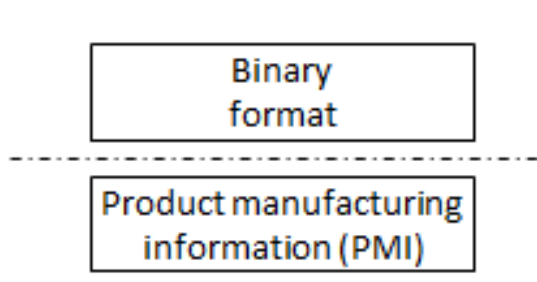

- Surface quality

- Welding points

- Welding lines

- Coordinate system

- Arbitrary primitive data type
Exact geometry through B-REP

- JT B-REP

- XT B-REP

Tessellation with different LOD ULP (Ultra-Lightweight Precise)

- Parts

- Attributes

Fig. 4 Contents in a JT file

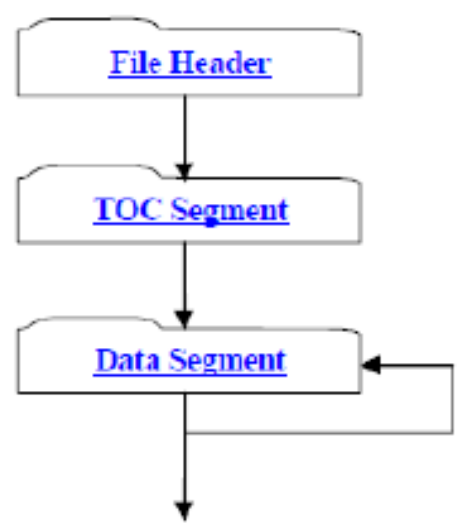

Fig. 5 Structure of a JT file

To view JT model in the browser, it is imperative to parse the information in JT file-according to the structure of JT file-into a format which can be parsed by browser. JSON (JavaScript Object Notation) is a lightweight data-interchange format not only easy for technicians to read and write, but also easy for program to parse and generate. It is a text format and can be parsed through JavaScript which runs on browser. Therefore it is a feasible method to parse the JT model into JSON files with java program. Information including filename, materials and vertices and so on in JT model can be stored in a JSON file as follows:

\{

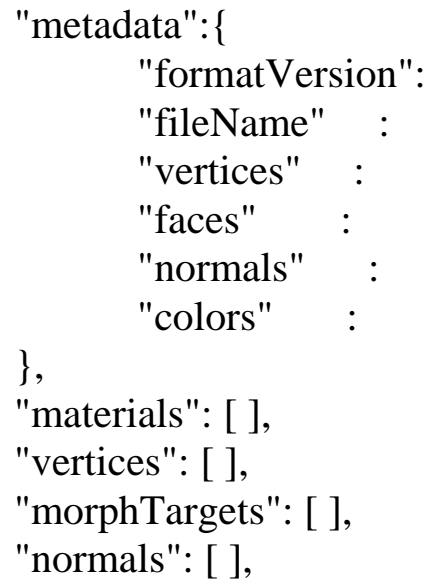




\section{"colors": [ ], \\ "uvs": [[ ]], \\ "faces": [ ] \\ \}}

Among these, filename is the name of part, which constitutes the assembly structural tree. Vertices and faces constitute the geometry of the 3D model. And material is a kind of information of PMI.

\subsection{Rendering Model in the Browser}

Rendering displays the model defined by geometry, material and so on into graphics. Graphics engine facilitates achieving the rendering process. A powerful graphics engine for visualization, rendering, and animation has become an important basic module of each modern CAD system. Nowadays, there is a vast array of 3D graphics engines such as Minko, OSG, OGRE, etc. which are respectively suitable for different occasions. Among these 3D graphics engines, three.js, a third-party library of WebGL written in JavaScript, is a 3D engine and runs in browser. Three.js provides lots of 3D display function and can be used to create all kinds of 3D scene, including the lighting, materials and other objects. On these accounts, three.js is utilized as graphics engine to render 3D model in the browser in this paper.

Three.js provides plenty of classes that can parse various 3D formats such as obj, stl, and text format like JSON. There is a class “THREE.JSONLoader" in three.js that can parse and render data in JSON files and it can be applied to load the JSON files generated in 3.2. To embed three.js in web pages, the following command is used:

$<$ script src $=$ "js/three.js" $></$ script $>$

Based on the procedures above, the overall process to implement the visualization of DMU in the browser is shown in Fig. 6.

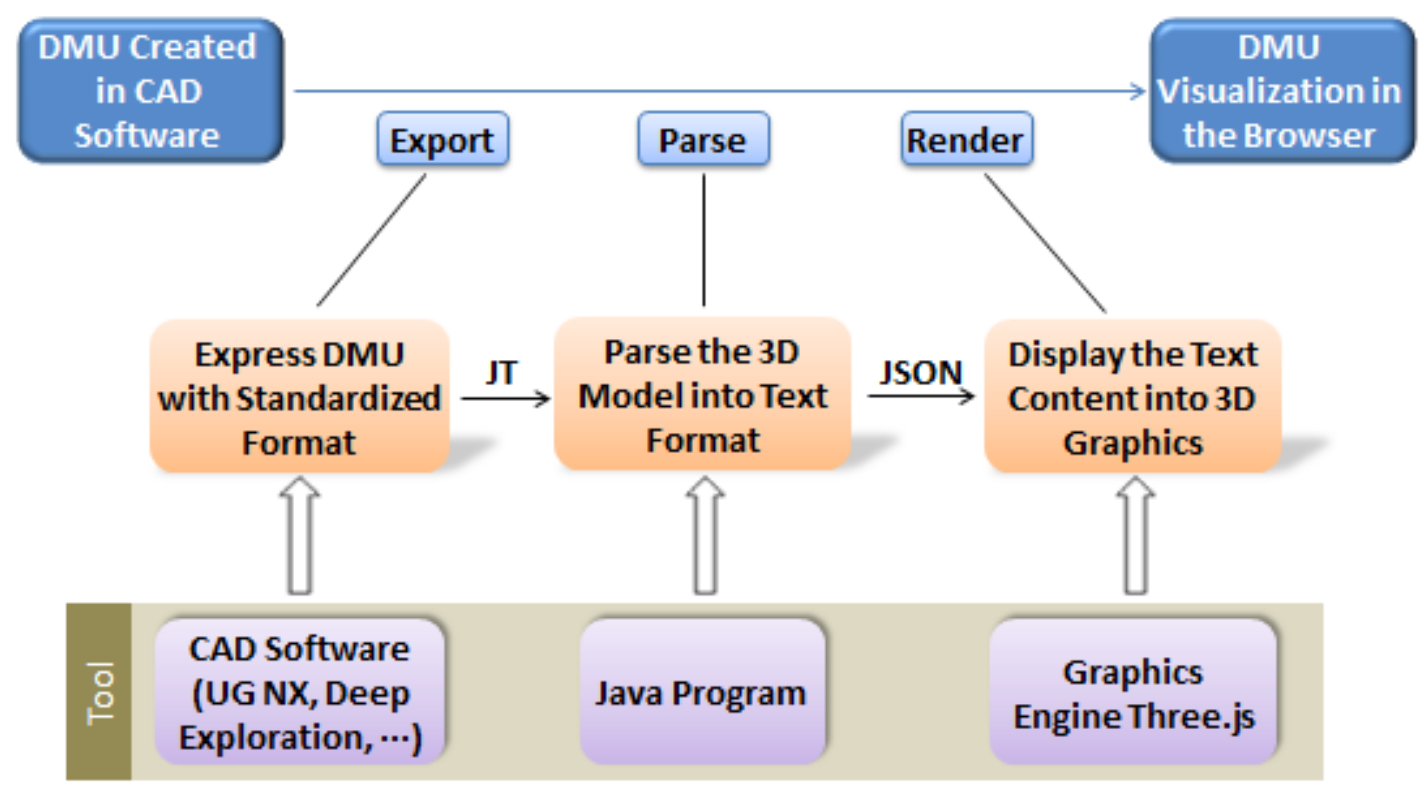

Fig. 6 Process of DMU visualization in the browser

\section{Case Study}

The method proposed above provides solution to accomplish the visualization of DMU in the browser, which is especially important for complex product development.

Aero-engine is a highly complex and precision product and requires collaboration between different teams during designing and manufacturing process. To improve collaborative efficiency, it is essential to have a quick access to the aero-engine based on web. A case of viewing and checking turbofan engine in the browser using the method proposed above is given in Fig. 7. The browser utilized in this case is google chrome. In the browser the assembly structural tree of the product is shown on the left. The original turbofan engine model is designed in UG NX, as shown in Fig. 8. 


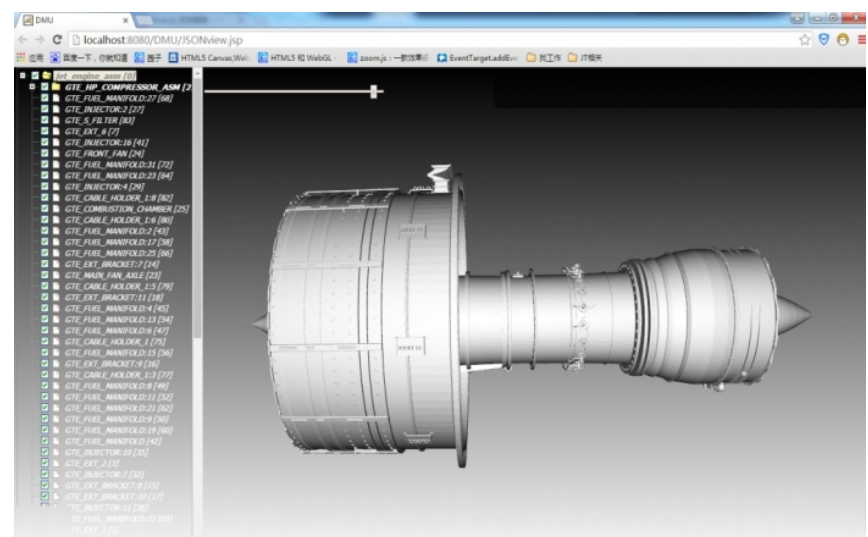

Fig. 7 The turbofan engine viewed in the browser

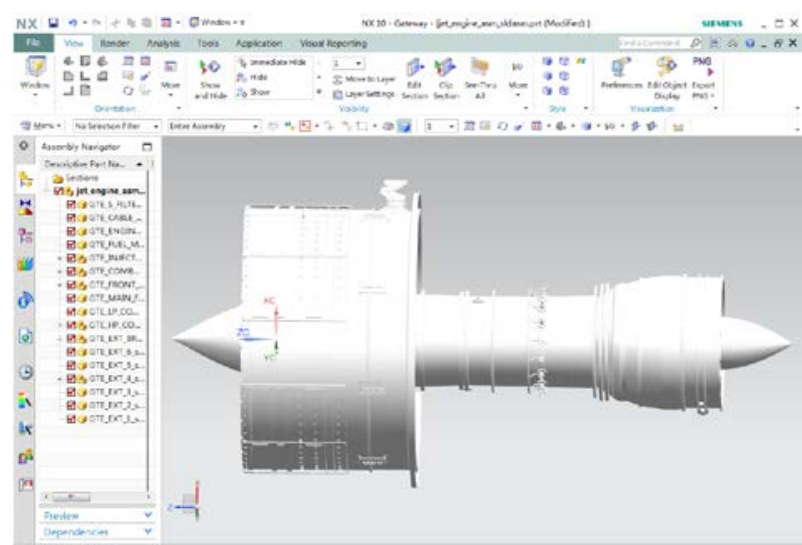

Fig. 8 The turbofan engine designed in UG NX

Interactive operation for 3D model in the browser is added in this case for the convenience of getting more information as needed. "TrackballControls.js" in three.js supports interaction between 3D scene and mouse operation. With the help of "TrackballControls.js", the 3D model can be viewed from every direction by rotation, translation and zooming in and out with the mouse, as shown in Fig. 9. Viewer can also click a part of the model with the mouse and the part will be highlighted in yellow, as shown in Fig. 10.

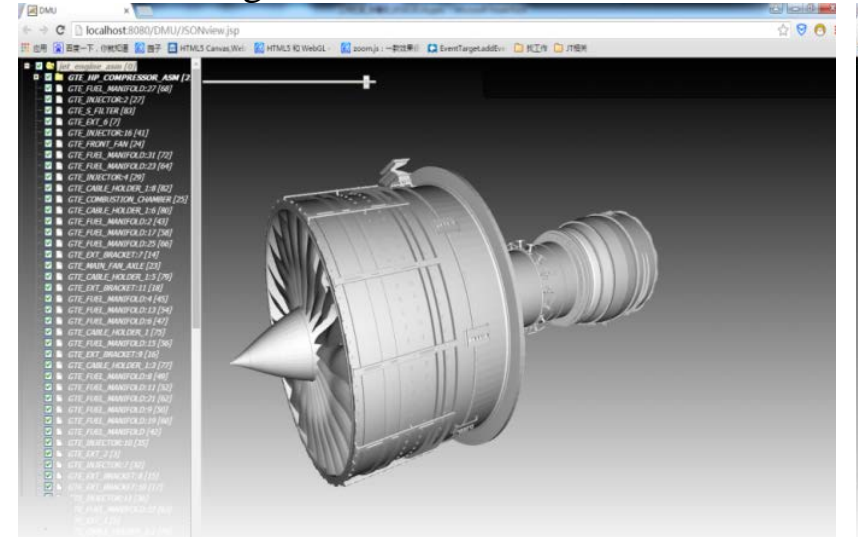

Fig. 9 Rotate or translate the model

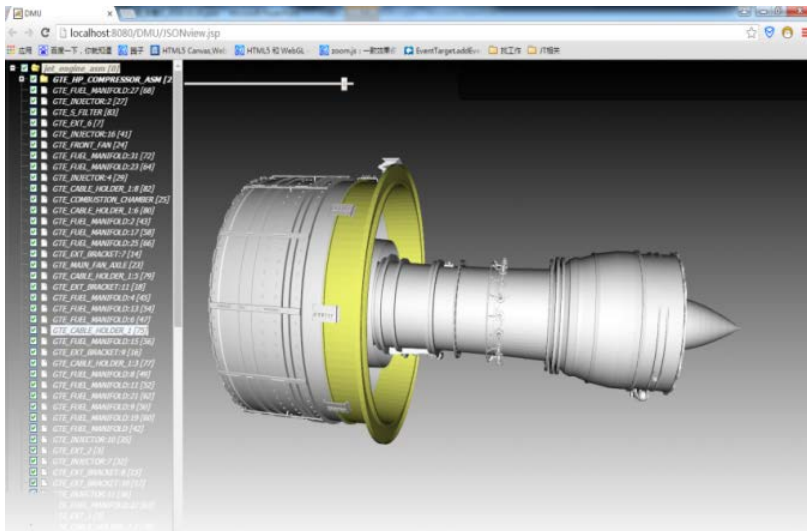

Fig. 10 Highlighting a part

\section{Summary}

The paper discusses how to accomplish the web based visualization of DMU with the standardized 3D visualization format JT. A practical method is introduced and a case of aero-engine using this method is given, which implements the visualization of aero-engine assembly model in the browser.

By the method proposed here, quick and precision construction of 3D collaborative platform for large-scale product model can be realized on the Internet, which means a more flexible and light-weighted working environment is possible for geographically distributed stakeholders in the same project. This method can be in common use and especially helpful for non-technical business apartments where the heavy CAD end is always expensive and resource-consuming, thus will facilitate the management and development activities greatly. While for exerting full potentials of DMU in web based environment, some future work still needs further efforts such as:

a) The definition and inclusion of more information. DMU is very complicated and a static model cannot exhibit all its advantages. It should be applied to implement further functionalities such as kinematics simulation in the browser.

b) Visualization is just the first step to exploit the DMU's capacity in work-floor practices. To combine with more manufacturing information and packaged with PLM or other management software will influence the manufacturing process in depth and change the prospect of future workshop. 
c) Once the 3D DMU on web can become the de facto standard for collaborative development in long term, it may provoke the update of CAD data definition theory and technology for that cooperative design offers a high-efficiency work pattern against the background of globalization.

\section{Acknowledgment}

This paper is sponsored by the National Science and Technology Major Project (2014ZX04001-081-07). The corresponding author of this paper is Yu Yong, and her email address is yuyong@buaa.edu.cn.

\section{References}

[1] Yong Hu. Research on Grid-based Virtual Reality Environment for Collaborative Review of Digital Mockups [D]. School of Mechanical Engineering Shanghai Jiao Tong University, 2013.

[2] Yang Z, Zhou J. Engineering portal with coordination mechanism for collaborative product development[C]// IEEE International Conference on Industrial Engineering and Engineering Management. IEEE, 2010:2263-2267.

[3] Yang Yunbin, Wang Fengjun, Wei Lifan. Study on Application of Digital Prototyping Technology in Engineering Design for Complex Product [J]. Machinery Design\&Manufacture, 2012(4):253-255.

[4] Sun G. A digital mock-up visualization system capable of processing giga-scale CAD models[J]. Computer-Aided Design, 2007, 39(2):133-141.

[5] Ding L, Ball A, Matthews J A, et al. DET2007 Special Issue: Annotation of lightweight formats for long-term product representations[J]. International Journal of Computer Integrated Manufacturing, 2009, 22(11):1037-1053.

[6] Mckenzieveal D. An Analysis of STEP, JT, and PDF Format Translation Between Constraint-based Cad Systems with a Benchmark Model[J]. Dissertations \& Theses - Gradworks, 2012.

[7] Emmer C, Fröhlich A, Stjepandic J. Advanced Engineering Visualization with Standardized 3D Formats[M]// Product Lifecycle Management for Society. Springer Berlin Heidelberg, 2013:286-295.

[8] Hartman N W, Lim A. Examining Neutral Formats for Visualization and Data Exchange[J]. Proc of the Iajc, 2008.

[9] International Organization for Standardization, 2012. Industrial automation systems and integration - JT file format specification for 3D visualization[J]. ISO 14306:2012.

[10] Ding L, Matthews J, Mcmahon C, et al. Product representation in lightweight formats for product lifecycle management (PLM)[J]. Opus, 2007.

[11]Eigner M, Handschuh S, Gerhardt F. Concept to Enrichen Lightweight, Neutral Data Formats with CAD-based Feature Technology[J]. Computer-Aided Design and Applications, 2013, 7(1):89-99.

[12]Handschuh S, Dotzauer R, Fröhlich A. Standardized Formats for Visualization: Application and Development of JT[M]// Concurrent Engineering Approaches for Sustainable Product Development in a Multi-Disciplinary Environment. Springer London, 2013:741-752.

[13] Stjepandić J, Wognum P M, Verhagen W. Concurrent Engineering in the 21st Century[M]// Concurrent Engineering in the 21st Century Foundations, Developments and Challenges. 2015. 
[14]Grimm M, Christ A, Anderl R. Distributed Additive Manufacturing - Concept for the Application of JT (ISO 14306) as Downstream Process Format[C]// ASME 2015 International Design Engineering Technical Conferences \& Computers and Information in Engineering Conference. 2015.

[15]Katzenbach A, Handschuh S, Vettermann S. JT Format (ISO 14306) and AP 242 (ISO 10303): The Step to the Next Generation Collaborative Product Creation[M]// Digital Product and Process Development Systems. Springer Berlin Heidelberg, 2013:41-52. 